\title{
Indicadores de sustentabilidade ambiental: métodos e aplicações
}

\author{
Indicators of environmental sustainability: methods and applications \\ Pedro Daniel da Cunha Kemerich', Luciana Gregory Ritter², Wilian Fernando Borba ${ }^{3}$ \\ ' Coordenador do Curso de Engenharia Ambiental da Universidade Federal de Santa Maria/CESNORS, Frederico Westphalen, Brasil. \\ ${ }_{2}^{2}$ Aluna de graduação do curso de Engenharia Ambiental e Sanitária, Universidade Federal de Santa Maria, Santa Maria, Brasil. \\ ${ }^{3}$ Técnico em Agropecuária, Aluno de Graduação do Curso de Engenharia Ambiental, Universidade Federal de Santa Maria, Santa Maria, \\ Brasil.
}

\section{Resumo}

Um instrumento utilizado para monitorar o desenvolvimento sustentável são os indicadores de sustentabilidade, os quais são responsáveis por capturar tendências para informar os agentes de decisão, orientar o desenvolvimento e o monitoramento de políticas e estratégias. Com base no exposto, o presente trabalho tem como objetivo apresentar uma síntese dos principais indicadores de sustentabilidade e suas aplicações. O modelo Pressão-Estado-Resposta evidencia os elos entre a atividade humana e o ambiente, porém reduz as pressões sobre o ambiente àquelas ocasionadas pelo homem. O modelo Força Motriz-Estado-Resposta estabelece um vínculo lógico entre os seus componentes, entretanto baseia-se na situação dos países industrializados. O modelo Força Motriz-Pressão-Estado-Impacto-Resposta reforça a interação entre as causas dos problemas ambientais, os impactos e as respostas da sociedade, porém ainda não existem experiências em medir a sustentabilidade em municípios, ou empreendimentos, já o Marco para a Avaliação de Sistemas de Manejo de Recursos Naturais incorporando Indicadores de Sustentabilidade possui ênfase nas avaliações qualitativas e quantitativas, no entanto sua base está assentada na comparação entre sistemas, sem definir isoladamente o que é sustentável ou insustentável, enquanto que a Pegada Ecológica mostra quanto da capacidade regenerativa da biosfera está sendo usada em atividades humanas, considerando apenas os efeitos econômicos referentes à utilização de recursos. Pode-se concluir que os indicadores de sustentabilidade, podem colaborar para a construção do desenvolvimento sob o enfoque integrador, considerando as dimensões sociais, ambientais e econômicas, para a consolidação de uma sociedade sustentável.

Palavras-chave: Modelo Pressão-Estado-Resposta. Sustentabilidade. Pegada Ecológica. Indicadores ambientais

\begin{abstract}
An instrument used to monitor sustainable development are sustainability indicators, which are responsible for capturing trends to inform decision-makers, guide the development and monitoring of policies and strategies. Based on the above, this paper aims to provide a summary of the key sustainability indicators and their applications. The model Pressure-State-Response highlights the links between human activity and the environment, but reduces those pressures on the environment caused by man. The model Driving Force-State-Response establishes a logical link between its components, however based on the situation in industrialized countries. The model Driving Force-Pressure-StateImpact-Response strengthens the interaction between the causes of environmental problems, impacts and responses of society, but there are still no experiences in measuring sustainability in cities, or developments since the Framework for systems Evaluation of Natural Resource Management incorporating Sustainability Indicators has an emphasis on qualitative and quantitative evaluations, however its base sits on a comparison between systems, without defining what is sustainable in isolation or unsustainable, while the Ecological Footprint shows how much capacity regenerative biosphere is being used in human activities, considering only the economic effects related to the use of resources. It can be concluded that indicators of sustainability, can collaborate to build the development under the integrative approach, considering the social, environmental and economic, to the consolidation of a sustainable society.
\end{abstract}

Keywords: Model Pressure-State-Response. Sustainability. Ecological Footprint. Environmental indicators. 


\section{INTRODUÇÃO}

A busca pelo equilíbrio entre o crescimento econômico e a manutenção dos recursos naturais tem nos últimos anos fortalecido o paradigma do desenvolvimento sustentável, e para tal tem-se procurado estabelecer mecanismos capazes de subsidiar as ações da sociedade que conduzam na direção do desenvolvimento sustentável (COUTO, 2007).

A raiz da grande polêmica referente ao desenvolvimento sustentável encontra-se em dois locais: nos indivíduos e no objetivo comum, isto é, em cada indivíduo além de uma noção e um conceito sobre desenvolvimento sustentável, há uma maneira implícita de como interagir, no processo, com o ambiente. A dificuldade em se estabelecer um objetivo conjunto, mesmo considerando as mudanças e pretensões individuais específicas, reside na forma como cada indivíduo percebe este "objetivo maior": uns acreditam que o objetivo almejado seja o desenvolvimento sustentável, outros pensam que se trata do próprio processo, e outros ainda, o percebem como um conjunto disso tudo (BENETTI, 2006).

A sustentabilidade é algo que não pode ser obtido instantaneamente, ela é um processo de mudança, de aperfeiçoamento constante e de transformação estrutural que deve ter a participação da população como um todo, e a consideração de suas diferentes dimensões (BENETTI, 2006).

A construção de um modelo de desenvolvimento, sob novas bases econômicas e em harmonia com a capacidade de suporte dos sistemas naturais, faz com que os agentes responsáveis por sua concepção necessitem de um amplo levantamento de dados e informações representativas das diversas dimensões envolvidas nos processos produtivos, bem como da condução de investigações que possibilitem um melhor entendimento dos sistemas ambientais (COUTO, 2007).

Para auxiliar nas avaliações sobre a sustentabilidade ambiental surgem os indicadores, cujo papel como ferramenta é o estabelecimento de uma visão de conjunto que exige um processo de avaliação de resultados em relação às metas de sustentabilidade estabelecidas, provendo às partes interessadas condições adequadas de acompanhamento e dando suporte ao processo decisório (MALHEIROS, PHILIPPI e COUTINHO, 2008).

Um indicador é um parâmetro, ou uma função derivada dele. Têm a capacidade de descrever um estado ou uma resposta dos fenômenos que ocorrem em um meio. Ou seja, um indicador representa uma forma de percepção da realidade que se dá através de um conjunto de dados representativos de parâmetros capazes de traduzir o estado de um ambiente (SANTOS, 2004).

É através das informações obtidas mediante a utilização dos indicadores que poderão ser subsidiadas as etapas de planejamento, implantação e acompanhamento das políticas de gestão ambiental voltadas ao uso racional dos recursos naturais e ao ordenamento das intervenções no meio ambiente.

Com base no exposto, o presente trabalho tem como objetivo apresentar uma síntese dos principais indicadores de sustentabilidade e suas aplicações.

\section{O USO DE INDICADORES DE SUSTEN- TABILIDADE AMBIENTAL}

Os indicadores ambientais começaram a ser utilizados durante a década de 70 e 80 , como resultado de esforços de governos e organizações internacionais na elaboração e divulgação dos primeiros Relatórios sobre o Estado do Ambiente, (FRANCA, 2001). Sendo o governo holandês o pioneiro na adoção de indicadores ambientais, em 1989, para avaliar os resultados da implementação do Plano de Política Ambiental Nacional (HAMMOND et al, 1995).

A busca por indicadores de sustentabilidade ambiental cresceu bastante durante a última década, particularmente em sua segunda metade, principalmente por parte de organismos governamentais, não-governamentais, institutos de pesquisa e universidades em todo o mundo. Muitas conferências já foram organizadas por entidades internacionais, bem como outras iniciativas de pesquisadores ligados a algumas instituições governamentais e/ou universitárias (MARZALL; ALMEIDA, 2000).

Apesar da realização de conferências organizadas por entidades internacionais, se tem muito pouco de concentro, devido ao fato de o tema ser relativamente novo para a comunidade acadêmica, sendo poucas também as publicações que abordam o tema. Os conceitos são novos e os resultados de pesquisa e experimentação, em grande parte dos casos, não estão ainda disponíveis, pois os trabalhos, em sua maioria, estão em andamento (MARZALL; ALMEIDA, 2000).

\section{I A função dos indicadores na avaliação da sustentabilidade ambiental}

Os indicadores de sustentabilidade são ferramentas utilizadas para auxiliar no monitoramento da operacionalização do desenvolvimento 
sustentável, sendo a sua principal função fornecer informações sobre o estado das diversas dimensões (ambientais, econômicas, socioeconômicas, culturais, institucionais, etc.) que compõem o desenvolvimento sustentável do sistema na sociedade (CARVALHO, J. et al., 2011).

A utilização de indicadores tem por objetivo reunir e quantificar informações de um modo que sua importância se destaque, simplificando informações sobre fenômenos complexos tentando melhorar com isso o processo de comunicação (VAN BELLEN, 2006). Os indicadores também têm sido utilizados como ferramenta padrão, auxiliando na compreensão das informações sobre fenômenos complexos, em diversos estudos nacionais e internacionais, pois permite verificar os impactos das ações humanas no ecossistema (SILVA, CORREIA E CÂNDIDO, 2010).

De acordo com Benetti (2006), um indicador é uma ferramenta desenvolvida para obter informações referentes a uma dada realidade, tendo como característica principal a capacidade de sintetizar um conjunto complexo de informações, restando apenas o significado essencial dos aspectos analisados. Hammond et al (1995), em seus estudos, desenvolveram uma pirâmide de informação (Figura 1), a qual tem por objetivo relacionar dados primários e indicadores.

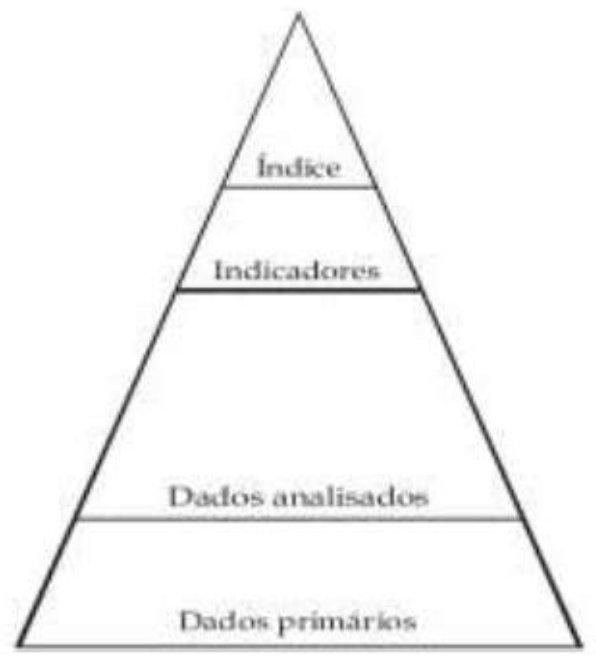

Figura 1 - Pirâmide de informações

Fonte: Hammond et al, 1995

Uma das grandes aplicações dos indicadores encontra-se na necessidade de monitoramento do progresso nas distintas dimensões, pois eles funcionam como ferramentas de apoio aos tomadores de decisões e àqueles responsáveis pela elaboração de políticas em todos os níveis, além de serem norteadores para que se mantenha o foco em direção ao desenvolvimento sustentável (GARCIA; GUERRERO, 2006). Servindo também como uma ferramenta para medir e avaliar as consequências das atividades antrópicas no sistema biológico (DANZ et al, 2005) bem como para permitir que as pessoas ajam sobre questões do meio ambiente (EEA, 1999).

Através da utilização de indicadores ambientais deve ser possível a análise das condições, mudanças da qualidade ambiental, além de favorecer o entendimento das interfaces da sustentabilidade, bem como de tendências, como uma ferramenta de suporte no processo de tomada de decisão e formulação de políticas e práticas sustentáveis (GOMES; MALHEIROS, 2012), pois os indicadores têm como função diagnosticar a saúde do ecossistema e fornecer uma ferramenta para monitorar condições e mudanças ambientais ao longo do tempo (JORGENSEN, 2005).

A partir da utilização dos indicadores de sustentabilidade, gera-se o Índice de Sustentabilidade, o qual é uma forma de sintetizar, matematicamente, uma série de informações quantitativas e semi-quantitativas, associadas à sustentabilidade do desenvolvimento. Cada índice, ao final, gerará um valor numérico, que será o resultado de operações matemáticas com as informações que utiliza, e, que quando comparado a uma escala padrão, avaliará a sustentabilidade (KRONEMBERGER et al., 2008).

Segundo a Organização das Nações Unidas (ONU) os indicadores não devem servir apenas aos interesses do Poder Público, para avaliar a eficiência e eficácia das políticas adotadas, mas devem servir também aos interesses dos cidadãos, tornando-se instrumento de cidadania, pois eles podem informar o estado do meio ambiente e da qualidade de vida (CÂMARA, 2002).

Entretanto, os indicadores não são e nem devem ser vistos como soluções para todas as dificuldades que envolvem a sustentabilidade, seja na sua avaliação ou na sua operacionalização. Fernandes (2004) afirma que o que deve ficar claro é que os indicadores cumprem com sua função, ou seja, simplesmente indicam os caminhos para avaliação, para a discussão e a percepção da sustentabilidade, cabendo a quem os utiliza realizar as demais etapas.

\subsection{A complexidade dos indicadores de sustentabilidade ambiental}

Observa-se que um dos maiores desafios enfrentados na quantificação ou qualificação da sustentabilidade consiste na elaboração de metodologias adequadas que permitam avaliar a sus- 
tentabilidade de realidades locais, regionais ou nacionais, posto existirem diferentes características e peculiaridades inerentes aos aspectos sociais, econômicos, ambientais, culturais e institucionais.

$\mathrm{Na}$ literatura existe uma grande variedade de parâmetros para inferir o desempenho de sistemas hídricos e ambientais - são os chamados 'indicadores'. No entanto, tem-se verificado uma grande dificuldade em agregá-los em um único parâmetro - o 'índice' - capaz de traduzir numericamente uma situação e apontar, ao tomador de decisão, o sentido da sustentabilidade da região (VIEIRA; STUDART, 2009).

Sendo considerados o principal componente da avaliação do progresso em relação a um desenvolvimento dito sustentável, os indicadores podem ser um instrumento adequado para compatibilizar o crescimento econômico com a preservação ambiental e justiça social, principalmente porque nestes sistemas de indicadores estão contidas todas as informações pertinentes à situação econômica, social, e ambiental de um espaço geográfico em um determinado período (LIRA, 2008).

Torna-se fundamental a atividade de planejamento para a definição de um indicador capaz de reduzir o número de parâmetros e medidas sobre o meio, pois este é um processo que exige agilidade e eficiência no emprego de recursos, sendo inaceitável a medição de todas as variáveis ambientais (SALDANHA, 2007).

Para Dahl (1997) o maior desafio dos indicadores é fornecer um retrato da situação de sustentabilidade, de uma maneira simples, apesar da incerteza e da complexidade. $\mathrm{O}$ autor ainda ressalta a diferença dos países, a questão da diversidade cultural e os diferentes graus de desenvolvimentos como importantes fatores na construção dos indicadores.

Outra dificuldade dos indicadores é que não existe a possibilidade de medir a sustentabilidade de uma determinada escala considerando apenas um indicador que se refira a apenas um aspecto, pois a sustentabilidade é determinada por um conjunto de fatores (econômicos, sociais, ambientais, culturais e institucionais) e todos devem ser contemplados simultaneamente. Dessa forma, ao se avaliar a sustentabilidade deve-se usar sempre um conjunto de indicadores (MARZALL; ALMEIDA, 1999).

A fim de que se alcance êxito na avaliação é importante a definição clara dos objetivos que devem ser alcançados pelo programa e pelos indicadores propostos. Também deve-se prezar pela qualidade do indicador, sendo que esta depende das propriedades dos componentes utilizados em sua formulação e da precisão dos sistemas de informação empregados. O grau de excelência de um indicador deve ser definido por sua validade, ou seja, sua capacidade de medir o que se pretende, por sua confiabilidade, capacidade de reproduzir os mesmos resultados quando aplicado em condições similares. Em geral, a validade de um indicador é

Tabela 1. Síntese de algumas vantagens e limitações da aplicação de indicadores de desenvolvimento sustentável.

\begin{tabular}{|c|c|}
\hline Vantagens & Limitações \\
\hline $\begin{array}{l}\text {-Avaliação dos níveis de } \\
\text { desenvolvimento sustentável. } \\
\text {-Capacidade de sintetizar a informação } \\
\text { de carácter técnico/científico; } \\
\text {-Identificação das variáveis-chave do } \\
\text { sistema; } \\
\text {-Facilidade de transmitir a informação; } \\
\text {-Bom instrumento de apoio à decisão e } \\
\text { aos processos de gestão ambiental; } \\
\text {-Sublinhar a existência de tendências; } \\
\text {-Possibilidade de comparação com } \\
\text { padrões e/ou metas pré-definidas. }\end{array}$ & $\begin{array}{l}\text {-Dificuldades na definição de expressões } \\
\text { matemáticas que melhor traduzam os } \\
\text { parâmetros selecionados; } \\
\text {-Perda de informação nos processos de } \\
\text { agregação dos dados; } \\
\text {-Diferentes critérios na definição dos limites de } \\
\text { variação do índice em relação às imposições } \\
\text { estabelecidas; } \\
\text {-Ausência de critérios robustos para seleção de } \\
\text { alguns indicadores; } \\
\text {-Dificuldades na aplicação em determinadas } \\
\text { áreas como o ordenamento do território e a } \\
\text { paisagem. }\end{array}$ \\
\hline
\end{tabular}

Fonte: DGA, 2000 


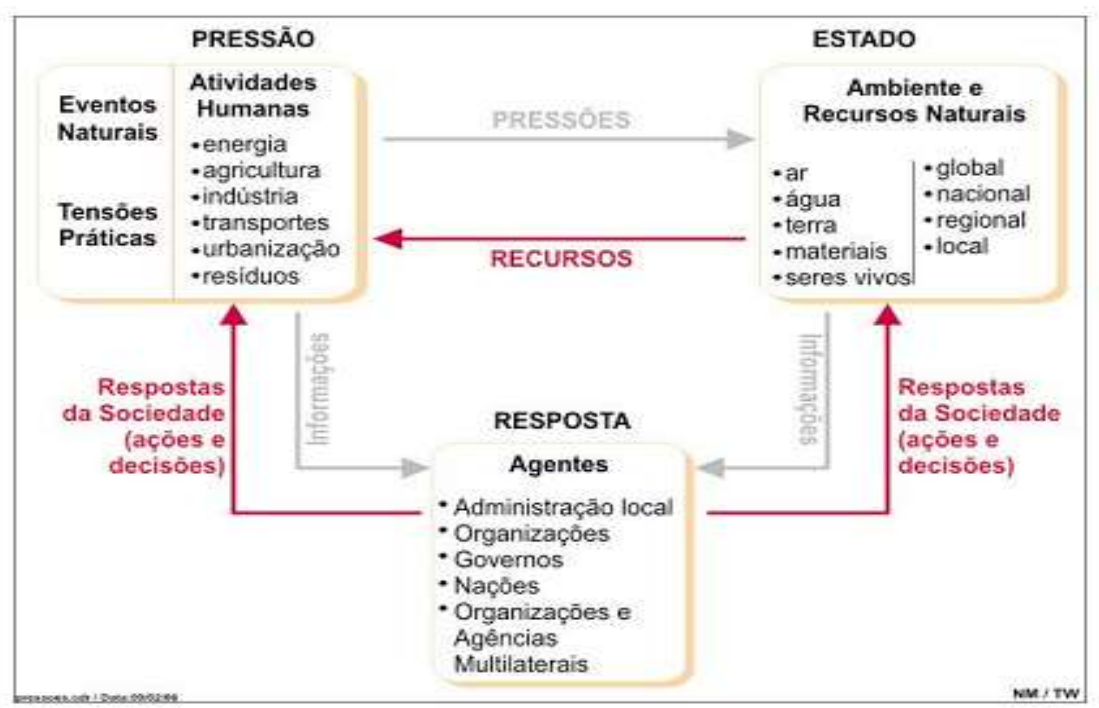

Figura 2 - Estrutura PER

Fonte: Maranhão, 2007

determinada pelas características de sensibilidade, capacidade de medir as alterações do fenômeno e especificidade, capacidade de medir somente o fenômeno analisado (SALDANHA, 2007).

Em função das simplificações que são efetuadas na aplicação dos indicadores, sempre há alguma controvérsia técnica/científica. As eventuais perdas de informação têm constituído um entrave à adoção de forma generalizada e consensual dos sistemas de indicadores. Na Tabela 1 apresenta-se uma síntese de algumas das principais vantagens e limitações da aplicação destes métodos.

\section{O MODELO PRESSÃO-ESTADO-RES- POSTA (PER)}

O método de Pressão-Estado-Resposta foi criado pela Organização para Cooperação e Desenvolvimento Econômico (OCDE), sendo baseado no conceito de causalidade, segundo o qual as atividades humanas exercem pressão sobre o ambiente alterando a qualidade e a quantidade de recursos naturais, ou seja, alterando o seu estado, e a sociedade responde a essas mudanças mediante políticas ambientais, econômicas ou setoriais (OCDE,1993).

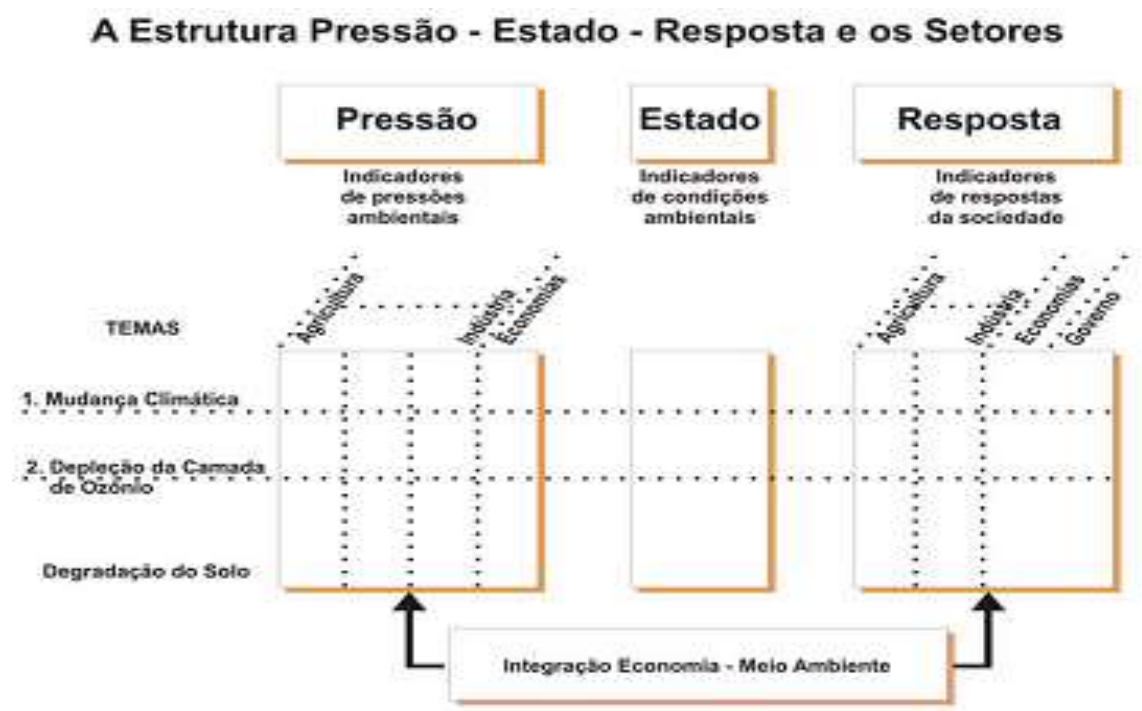

Figura 3 - A estrutura PER e articulação com temas específicos

Fonte: Maranhão, 2007 
De acordo com a OCDE (1993), o método PER apresenta a vantagem de evidenciar os elos entre a atividade humana e o ambiente e, ajudar os tomadores de decisão e o público a perceber a interdependência entre as questões ambientais e as outras (sem, todavia, esquecer que existem relações mais complexas nos ecossistemas e nas interações meio ambiente-sociedade).

Nele, os indicadores são divididos em três categorias (LIRA, 2008):

- Indicadores da pressão ambiental - descrevem as pressões das atividades humanas sobre o ambiente, incluindo a quantidade e qualidade dos recursos naturais;

- Indicadores das condições ambientais ou de estado - referem-se à qualidade do ambiente e à qualidade e quantidade dos recursos naturais. Eles devem fornecer uma visão da situação do ambiente e sua evolução no tempo, não das pressões sobre ele; - Indicadores das respostas sociais - são medidas que mostram a resposta da sociedade às mudanças ambientais, podendo estar relacionadas à prevenção dos efeitos negativos da ação do homem sobre o ambiente, à paralisação ou reversão de danos causados ao meio, e à preservação e conservação da natureza e dos recursos naturais.

A Figura 2 apresenta diagramaticamente a estrutura Pressão - Estado - Resposta, exibindo seus componentes e as relações existentes entre eles.

A Figura 3 permite visualizar como a estrutura conceitual PER pode articular-se com os setores da economia ou com temas e subtemas e, dessa forma, ganhar em detalhe.

Com objetivo de melhor integrar os aspectos ambientais às políticas setoriais, a OECD (1993) procurou agrupar os indicadores por temas e por setores. A classificação por temas é dividida em: mudança climática, diminuição da camada de ozônio, eutrofização, acidificação, contaminação tóxica, qualidade ambiental urbana, biodiversidade, paisagens culturais, resíduos, recursos hídricos, recursos florestais, recursos pesqueiros, degradação do solo (desertificação e erosão) e indicadores gerais. Os setores são classificados em transportes, energia e agricultura (LIRA; CÂNDIDO, 2008).

O modelo PER possibilita uma visão conjunta dos vários componentes de um problema ambiental, sendo esta uma grande vantagem que facilita o diagnóstico do problema e a elaboração da respectiva política pública, pois vai além da mera constatação da degradação ambiental e revela seu impacto, suas causas, o que está por trás dessas causas e as ações que estão sendo tomadas para melhorar esse quadro (CARVALHO, P., 2007).

Entretanto, este modelo reduz as pressões sobre o ambiente àquelas causadas pela ação do homem, desconsiderando as provenientes da ação da natureza, e sabe-se que os eventos naturais também podem causar impacto ambiental, sendo fontes de pressão (FERREIRA; LIRA; CÂNDIDO, 2010).

De acordo com Martinez (2001), a principal crítica a esses modelos é que induzem a leitura da existência de uma relação de causalidade linear, o que causa uma simplificação excessiva de uma situação complexa que envolve causalidades múltiplas e interação de fenômenos sociais, econômicos e ambientais. A adoção do modelo PER acabaria, portanto, estimulando a adoção de políticas corretivas, de curto prazo. Esse modelo também não estabelecem metas de sustentabilidade a serem

Tabela 2. Síntese de algumas potencialidades e fragilidades do modelo PER

\begin{tabular}{|c|c|}
\hline \multicolumn{2}{|c|}{ Modelo Pressão-Estado-Resposta (PER) } \\
\hline Potencialidades & Fragilidades \\
\hline $\begin{array}{l}\text {-Evidencia os elos entre a atividade } \\
\text { humana e o ambiente. }\end{array}$ & $\begin{array}{l}\text {-Pressões sobre o ambiente são reduzidas } \\
\text { àquelas causadas pela ação do homem. }\end{array}$ \\
\hline $\begin{array}{l}\text {-Visão conjunta } \text { dos } \\
\text { componentes de um prios } \\
\text { ambiental. }\end{array}$ & $\begin{array}{l}\text {-Existência de uma relação de } \\
\text { causalidade linear, a qual simplifica } \\
\text { excessivamente uma situação complexa. } \\
\text {-Não estabelece metas de sustentabilidade } \\
\text { a serem alcançadas. }\end{array}$ \\
\hline
\end{tabular}


alcançadas e como foi concebido originalmente para tratar de indicadores ambientais, nem sempre é adaptável para indicadores de desenvolvimento sustentável (IDS) onde a complexidade é maior.

$\mathrm{Na}$ Tabela 2 apresenta-se uma síntese das principais potencialidades e fragilidades do modelo PER.

A Comissão de Desenvolvimento Sustentável da ONU abandonou o modelo PER em 1999 com o argumento de que o mesmo não enfatizava os temas centrais das políticas públicas (IBGE, 2002).

\section{O MODELO FORÇA MOTRIZ-ESTADO- RESPOSTA (F-E-R)}

O Departamento de Coordenação Política e Desenvolvimento Sustentável (Department for Policy Coordination and Sustainable Development - DPCSD) da Divisão das Nações Unidas para o Desenvolvimento Sustentável propôs a classificação dos indicadores segundo o modelo Força Motriz-Estado-Resposta, adaptado do modelo Pressão-Estado-Resposta, como uma proposta de desenvolvimento de indicadores para o monitoramento do desenvolvimento sustentável de países mediante a implementação da Agenda 21. O termo força motriz, na visão do DPCSD seria mais apropriado para reunir indicadores econômicos, sociais e institucionais. Nesse caso, os indicadores das forças motrizes descrevem as atividades humanas, processos e padrões de impacto sobre o desenvolvimento sustentável (LIRA; CÂNDIDO, 2008).

Este modelo busca estabelecer um vínculo lógico entre os seus componentes, a fim de avaliar o estado do meio ambiente a partir dos fatores que exercem pressão (a força motriz) sobre os recursos naturais, do estado resultante destas pressões e das respostas que são produzidas para enfrentar esses problemas ambientais (PHILIPPI JR, MALHEIROS, AGUIAR, 2005).

Na Tabela 3 apresenta-se uma síntese das potencialidades e fragilidades do modelo FER.

\subsection{O modelo Força Motriz-Pressão-Estado- Impacto-Resposta (Driving Force-Pressure-State- Impact-Response)}

De acordo com Lira (2008), o modelo DPSIR (Forças motoras, Pressões, Estado, Impacte e Respostas) deriva do modelo PER (Pressão-Estado- Resposta) e foi adaptado pela OCDE a fim de que fosse possível perceber as ligações existentes entre o ambiente através dos indicadores ambientais e a sociedade através do desempenho ambiental. Este modelo tem por objetivo fornecer informação sobre os diferentes elementos da cadeia DPSIR, demonstrar a sua interligação e avaliar a eficácia das respostas.

Este modelo considera que as atividades econômicas e o comportamento humano afetam a qualidade ambiental. No entanto, as relações entre estes fenômenos são complexas. O modelo DPSIR reforça a interação entre as causas dos problemas ambientais, os impactos e as respostas da sociedade, de uma forma integrada (LIRA, 2008).

A Figura 4 apresenta graficamente a estrutura DPSIR e os seus componentes.

Para Soares et al. (2008), o modelo DPSIR define valores as atividades humanas responsáveis por gerar pressões (forças motrizes) e considera os elementos do impacto no ambiente, que exigem ações de respostas nos diferentes setores (ações políticas e macroeconômicas). E ainda, avalia as interações do meio ambiente com o desenvolvimento social e econômico. Sendo assim, na Tabela 4 apresenta-se uma síntese das potencialidades e fragilidades do modelo DPSIR.

Tabela 3. Síntese de algumas potencialidades e fragilidades do modelo FER

\begin{tabular}{lll}
\hline \multicolumn{2}{c}{ Modelo força motriz-estado-resposta (F-E-R) } \\
\hline Potencialidades & Fragilidades \\
\hline -Mais apropriado para reunir & & - os indicadores se baseiam na situação \\
indicadores econômicos, sociais e & dos países industrializados. \\
institucionais. & \\
-Estabelece um vínculo lógico entre os \\
seus componentes. \\
- Não existe nenhuma causalidade \\
entre os indicadores.
\end{tabular}




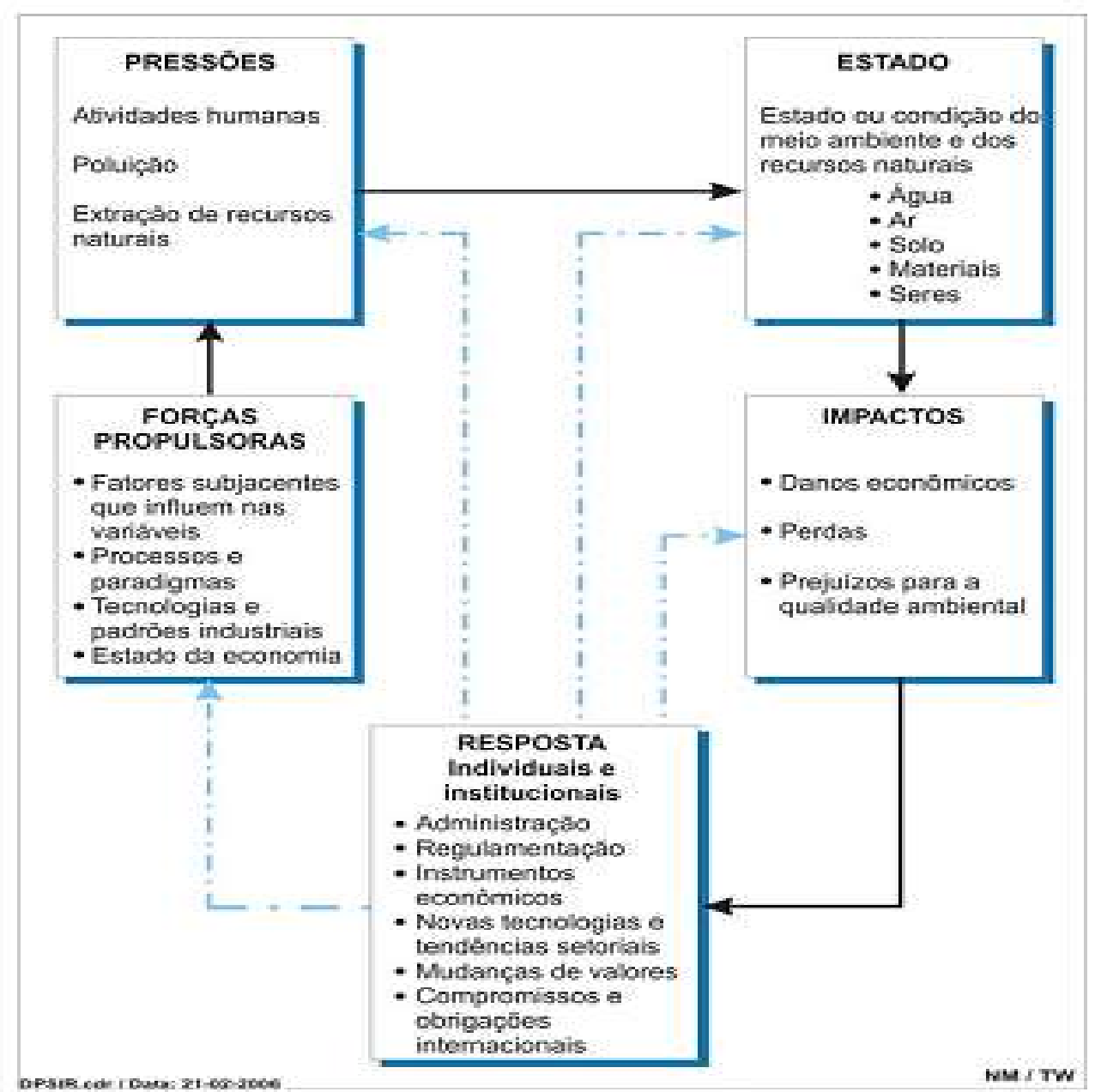

Figura 4 - A estrutura DPSIR

Fonte: Maranhão, 2007

Tabela 4. Síntese de algumas potencialidades e fragilidades do modelo DPSIR

\begin{tabular}{lll}
\hline \multicolumn{2}{c}{ Modelo Força Motriz-Pressão-Estado-Impacto-Resposta (DPSIR) } \\
\cline { 1 - 2 } \cline { 1 - 1 } - Reforça a interação entre as causas & & -muito utilizado em países com limites \\
dos problemas ambientais, os impactos & & geográficos nacionais, mas não existem \\
e as respostas da sociedade, de uma & & $\begin{array}{l}\text { experiências em medir a sustentabilidade } \\
\text { formanicípios, ou empreendimentos. }\end{array}$ \\
forma integrada. & & \\
-Considera os elementos do impacto & \\
no ambiente, que exigem ações de & \\
resposta & & \\
nos diferentes setores (ações políticas e \\
macroeconômicas) \\
-Permite o acompanhamento das \\
relações sociedade-meio ambiente e \\
comparaçôes nos planos nacional e \\
internacional.
\end{tabular}




\section{O MARCO PARA A AVALIAÇÃO DE SISTEMAS DE MANEJO DE RECURSOS NATURAIS INCORPORANDO INDICA- DORES DE SUSTENTABILIDADE (MES- MIS)}

Outro método para seleção de indicadores é o Marco para a Avaliação de Sistemas de Manejo de Recursos Naturais incorporando Indicadores de Sustentabilidade (MESMIS), um marco metodológico que objetiva avaliar a sustentabilidade de diferentes sistemas de manejo de recursos naturais em uma escala local.

O método MESMIS de avaliação de sustentabilidade, que em sua estrutura faz uso de Indicadores de Sustentabilidade, possui uma série de características que devem ser ressaltadas. Primeiramente, a proposta trata-se de um processo que permite adaptações de acordo com as necessidades específicas dos agroecossistemas que estão sendo avaliados. Destaca-se ainda, que é um exercício onde é essencial a valorização da participação de todos os atores e é, sem dúvida, um trabalho interdisciplinar. Finalmente, destaca-se pela exigência da abordagem das dimensões ambientais e socioeconômicas, dando ênfase às avaliações qualitativas e quantitativas (VERONA, 2010).

$\mathrm{Na}$ estrutura MESMIS, a avaliação não é concebida como um processo linear, mas como uma espiral de sucessivas avaliações. As conclusões e recomendações obtidas formam o ponto de partida de um novo ciclo, mostrando-se útil para sistematizar experiências, discutir sobre fortalezas e debilidades dos sistemas (ASTIER, 2004).

$\mathrm{O}$ método MESMIS tem sido utilizado como uma das ferramentas para a avaliação de sustentabilidade de agroecossistemas. Ele fornece bases conceituais e metodológicas para operacionalizar a sustentabilidade de agroecossistemas em âmbito local por meio da definição, medição e monitoramento de indicadores, partindo de uma abordagem sistêmica, interdisciplinar e participativa (MASER, ASTIER, LÓPEZ-RIDURA, 2000; SPEELMAN et al., 2007).

Esta metodologia é uma das mais completas, pois ela parte da definição do objeto de análise, seleciona indicadores, realiza a avaliação e o monitoramento e, ainda, recomenda mudanças e ajustes quando necessário (PEREIRA; MARTINS, 2010).

Verona (2010) diz que, no caminho da avaliação da sustentabilidade de um agroecossistema o método MESMIS propõe o seguimento das seguintes fases:

1- Estudo detalhado dos agroecossistemas da proposta de avaliação, identificando os sistemas de manejo, suas características e contexto socioeconômico e ambiental; 2- Análise dos pontos críticos existentes nos agroecossistemas: tratando de identificar os fatores limitantes e positivos relacionados com a sustentabilidade;

3- Seleção de indicadores: nesta etapa são determinados os critérios de diagnósticos, a partir dos quais derivam os indicadores estratégicos com os quais são realizadas as avaliações. Estes indicadores podem dar origem aos indicadores de sustentabilidade compostos, no caso da necessidade do uso destes.

4- Mensuração dos indicadores através da formulação de instrumentos de avaliação, com o objetivo de obter, quantificar, as informações desejadas, de origens qualitativas e quantitativas;

5- Apresentação e integração dos resultados: neste passo as avaliações quantitativas e qualitativas são passadas para valores numéricos, comumente com o uso de tabelas de notas. Os resultados encontrados na avaliação dos agroecossistemas são analisados e discutidos com todos os atores. Podem ser utilizadas técnicas estatísticas com testes multivariados como o uso da Análise de Componentes Principais e Análise Hierárquica de Agrupamentos. Outra ferramenta que pode ser utilizada é a Análise Multicritério. Para facilitar a visualização dos resultados no aspecto mais amplo, geralmente são construídos gráficos tipo radial (ameba). Nesta etapa, são abordados os principais obstáculos para a sustentabilidade, assim como os aspectos que mais a favorecem;

6- Indicações gerais para os agroecossistemas: nesta última etapa é realizada uma síntese da avaliação e são propostas alternativas para fortalecer a sustentabilidade dos sistemas de manejo, assim como para melhorar o processo da própria avaliação em trabalhos futuros (VERONA, 2010).

As fases acima descritas encontram-se ilustradas na figura 5. Cabe ressaltar que o denominado "tempo 2" onde é proporcionada a continuidade da avaliação de sustentabilidade, já em uma nova proposta de agroecossistema. Esta avaliação ao longo do tempo é denominada de "avaliação horizontal", pois para a avaliação continuada em anos subsequentes, o produto da última etapa serve de ponto inicial para o reinício do novo ciclo (PEREIRA; MARTINS, 2010). 


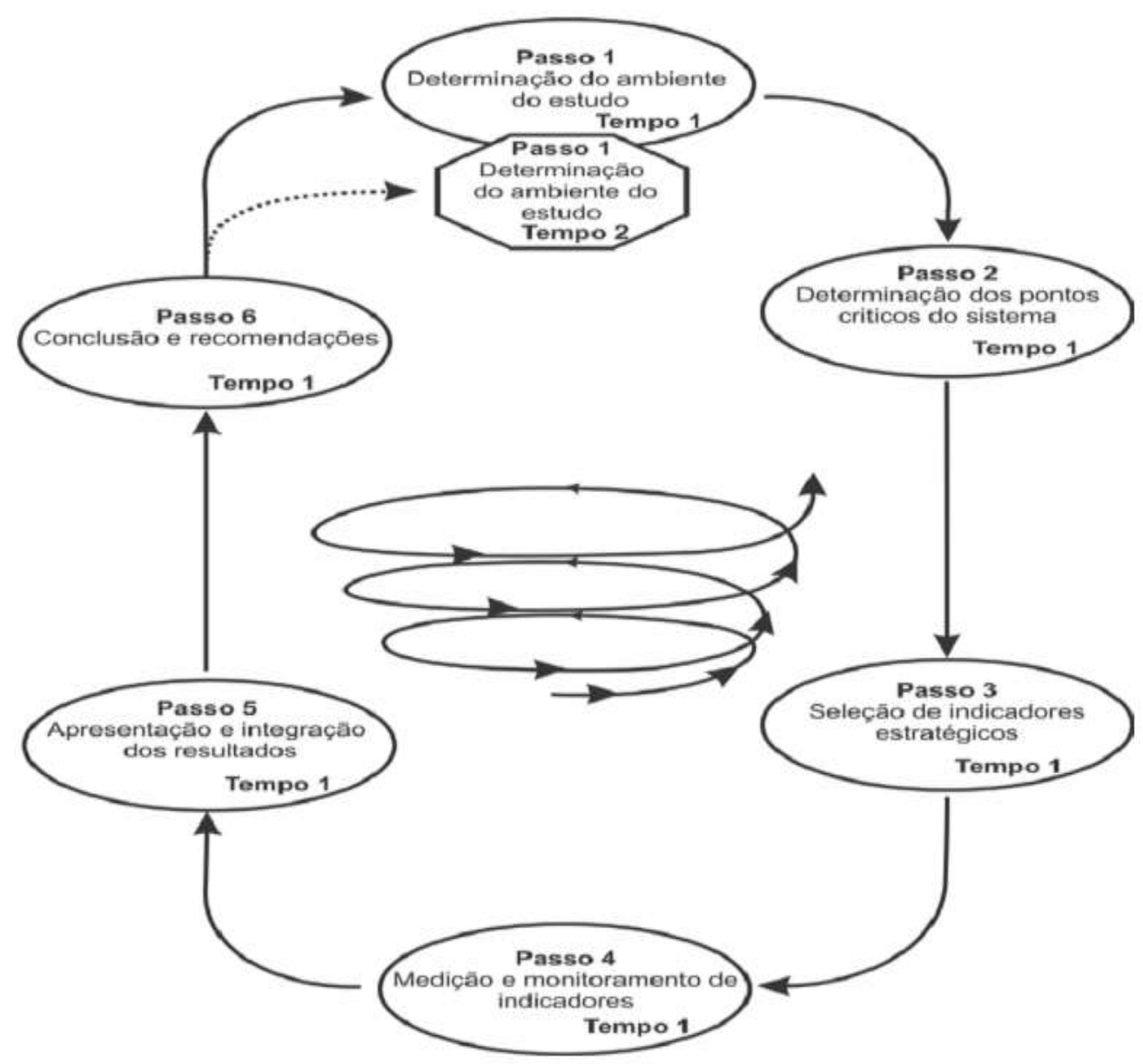

Figura 5 - Ciclo de avaliação da sustentabilidade pelo método MESMIS

Fonte: Verona, 2010

Destaca-se que a base do MESMIS está assentada na comparação entre sistemas, sem definir isoladamente o que é sustentável ou insustentável, pois salienta que é impossível obter uma medida absoluta de sustentabilidade, por isso faz uso de juízo do tipo "este sistema é mais (ou menos) sustentável de que este outro" (MASERA; ASTIER; LÓPEZ-RIDAURA, 2000).

$\mathrm{Na}$ Tabela 5 apresenta-se uma síntese das potencialidades e fragilidades do método MESMIS.

\section{PEGADA ECOLÓGICA}

O ecological footprint ou Pegada Ecológica (segundo a tradução brasileira) é um indicador que surgiu com o lançamento da obra "Our ecological footprint" de autoria de Wackernagel e Ress (2001), sendo este um trabalho pioneiro acerca do tema. A pegada ecológica pode ser definida como "a área de território ecologicamente produtivo necessária para produzir os recursos utilizados e para assimilar os resíduos produzidos por uma população da com um modo de vida especifico de forma indefinida" (WACKERNAGEL ET AL., 1997). O objetivo fundamental consiste em avaliar os impactos sobre o planeta de um determinado modo de vida e, consequentemente, seu grau de sustentabilidade.

O fundamento teórico da pegada ecológica relaciona-se com a capacidade de carga que é definida como a capacidade máxima de população que um sistema pode suportar de maneira indefinida no mesmo sistema (VAN BELLEN, 2006).

A Pegada Ecológica não envolve os malabarismos exigidos pelas abordagens monetárias, além de transmitir uma noção que pode ser facilmente assimilada pelo público preocupado em encontrar bons indicadores de sustentabilidade. Só pretende mostrar quanto da capacidade regenerativa da biosfera está sendo usada em atividades humanas (consumo). 
Tabela 5. Síntese de algumas potencialidades e fragilidades do método MESMIS

O Marco para a Avaliação de Sistemas de Manejo de Recursos Naturais incorporando Indicadores de Sustentabilidade (MESMIS)

\begin{tabular}{ll}
\hline \multicolumn{2}{c}{ O Marco para a Avaliação de Sistemas de Manejo de Recursos Naturais } \\
incorporando Indicadores de Sustentabilidade (MESMIS)
\end{tabular}

Fonte: Elaborada pelos autores

Versões mais recentes da metodologia de cálculo da pegada enfatizam que o superávit ecológico de uma nação não pode ser entendido como critério de sustentabilidade. Mais do que isso, autores da metodologia passaram a insistir que a pegada de cada país seja comparada à biocapacidade global em vez da nacional (MORAN et al., 2008). E isso obriga, então, que a pegada ecológica seja entendida como um indicador da contribuição dada à insustentabilidade global, em vez de um indicador de sustentabilidade deste ou daquele país, região ou localidade.

Uma outra limitação, segundo Hardi e Barg (1997), refere-se ao fato de o sistema ser estático, não permitindo extrapolações no tempo. Os resultados refletem um estado atual e a ferramenta não pretende fazer extrapolações, apenas sensibilizar a sociedade. O sistema também não inclui diversas questões importantes, que muitas vezes estão diretamente relacionadas à utilização da terra, como áreas perdidas de produtividade biológica em função de contaminação, erosão e utilização

Tabela 6. Síntese de algumas potencialidades e fragilidades do indicador Pegada Ecológica

\begin{tabular}{|c|c|}
\hline \multicolumn{2}{|c|}{ Pegada Ecológica } \\
\hline Potencialidades & Fragilidades \\
\hline $\begin{array}{l}\text {-Avaliar os impactos sobre o planeta } \\
\text { de um determinado modo de vida e, } \\
\text { consequentemente, seu grau de } \\
\text { sustentabilidade. }\end{array}$ & $\begin{array}{l}\text {-O superávit ecológico de uma nação } \\
\text { não pode ser entendido como critério de } \\
\text { sustentabilidade. }\end{array}$ \\
\hline
\end{tabular}

-Reforça a necessidade de introduzir a questão da capacidade de carga na sociedade.

-O sistema é estático, não permitindo extrapolações no tempo.

-Mostra quanto da capacidade regenerativa da biosfera está sendo usada em atividades humanas.

-Apenas considera os efeitos econômicos das decisões relativas à utilização de recursos. 
urbana. O Ecological Footprint Method apenas considera os efeitos econômicos das decisões relativas à utilização de recursos. Estas simplificações na metodologia de cálculo muitas vezes levam a perspectivas mais otimistas do que efetivamente ocorre na realidade.

$\mathrm{Na}$ Tabela 6 apresenta-se uma síntese das potencialidades e fragilidades do indicador pegada ecológica.

\section{CONSIDERAÇÕES FINAIS}

Ao analisar alguns dos principais indicadores de sustentabilidade ambiental é possível observar os seus pontos fracos e fortes, sendo que as discussões sobre este aspecto auxiliam na escolha do indicador mais adequado para a avaliação em questão, auxiliando na tomada de decisões referentes aos questionamentos da sustentabilidade.

Os indicadores de sustentabilidade ambiental devem possibilitar uma visão de conjunto, necessitando para tal, serem construídos a partir dos problemas e da realidade existente, a fim de que se possa entender seus aspectos críticos e usufruir de seu verdadeiro potencial.

Deve-se buscar o desenvolvimento, mas sob um enfoque integrador, mediante a utilização dos indicadores, considerando as dimensões sociais, ambientais e econômicas, para a consolidação de uma sociedade sustentável.

\section{REFERÊNCIAS}

ASTIER, M. La evaluación de la sustentabilidad em los sistemas de manejo: el Proyecto MESMIS. In: Congresso Brasileiro de Agroecologia, 1. Seminário Internacional sobre Agroecologia e V Seminário Estadual de Agroecologia, 4. 2003. Porto Alegre, RS. Anais... . Emater/RS-Ascar, Porto Alegre; Embrapa Clima T emperado, Pelotas, 2004,p. 234-240.

BENETTI, L. B. Avaliação do índice de desenvolvimento sustentável do município de Lages (SC) através do método do Painel de Sustentabilidade. 2006. 215f. Tese (Doutorado em Engenharia Ambiental) Curso de Pós-Graduação em Engenharia Ambiental, Universidade Federal de Santa Catarina, 2006.

BROWN, L.; FLAVIN, C.; FRENCH, H. Estado do mundo 2000. Tradução H. Mallett. Salvador: UMA Editora, 2000. 288p.
CÂMARA, J. B. D. (Org). GEO BRASIL 2002:

Perspectivas do Meio Ambiente no Brasil. Programa das Nações Unidas para o Meio Ambiente PNUMA. Edições IBAMA, Brasília, 2002.

CARVALHO, J. R. M. de; CURI, W. W. F; CARVALHO, E. K. M. de A, CURI, R. C. Proposta e validação de indicadores hidroambientais para bacias hidrográficas: estudo de caso na sub-bacia do alto curso do Rio Paraíba, PB. Revista Sociedade e Natureza, Uberlândia, v. 23, n. 2, agosto 2011.

CARVALHO, P. G. M. de; BARCELLOS, F. C; MOREIRA, C. G. Políticas públicas para meio ambiente na visão do gestor ambiental- Uma aplicação do modelo PER para o Semi-Árido. "VII Encontro da Sociedade Brasileira de Economia Ecológica" - Fortaleza, 28 a 30 de novembro de 2007.

COUTO, O. F. V. Geração de um índice de sustentabilidade ambiental para bacias hidrográficas em áreas urbanas através do emprego de técnicas integradas de geoprocessamento. 2007. Dissertação de Mestrado - Instituto de Geociências. Universidade Federal do Rio Grande do Sul. Porto Alegre, 2007.

DAHL, A. L. The big picture: compehensive approaches. In: MOLDAN G.; BILHARZ, S. (Eds.) Sustainability indicators: report of the project on indicators of sustainable development. Chichester: John Wiley \& Sons Ltd., 1997.

DANZ, N. P., et al. Environmentally stratified sampling design for the development of great lakes environmental indicators. Environmental Monitoring and Assessment, New York, n. 102, 2005, p. 41-65.

\section{DIREÇÃO GERAL DO AMBIENTE - DGA,} 2000. Proposta para um sistema de indicadores de desenvolvimento sustentável. Amadora, Portugal, 2000 .

EEA - European Environment Agency. (1999). Environmental indicators: typology and overview. Copenhagen: EEA. (Technical report, n.25).

FERNANDES, L. A. de O. The Meaning of Sustainability: Searching for Agrienvironmental Indicators. Manchester: University of Manchester - Institute for development policy and management, 2004. (Doctoral thesis).

FERREIRA, E. da S; LIRA, W. S; CÂNDIDO, G. A. Sustentabilidade no setor de mineração: uma apli- 
cação do Modelo Pressão-Estado-Impacto-Resposta. Engenharia Ambiental - Espírito Santo do Pinhal, v. 7, n. 3, p. 074 - 091, jul./set. 2010.

FRANCA, L. P. Indicadores ambientais urbanos: revisão da literatura. Parceria 21, 2001.

FREITAS, M. A. V. de; SANTOS, A. H. M. Importância da água e da informação hidrológica. In: Freitas, M.A.V. de. (Ed.). O estado das águas no Brasil; perspectivas de gestão e informações de recursos hídricos. Brasília: ANEEL/MME/MMA-SRH/ OMM, 1999, p.13-16.

GARCIA, S; \& GUERRERO, M. Indicadores de sustentabilidad ambiental en La gestión de espacios verdes: Parque urbano Monte Calvário, Tandil, Argentina. Rev. geogr. Norte Gd., jul. 2006, no.35, p.45-57.

GOMES, P. R; MALHEIROS, T. F. Proposta de análise de indicadores ambientais para apoio na discussão da sustentabilidade. Revista Brasileira de Gestão e Desenvolvimento Regional, Taubaté, v. 8, n. 2, p. 151-169, mai-ago/2012.

HAMMOND, A.; ADRIAANSE, A.; RODENBURG, E.; BRYANT, D.; WOODWARD, R. Environmental indicators : a systematic approach to measuring and reporting on environmental policy performance in the context of sustainable development. Washington: World Resources Institute, 1995.

HARDI, P., BARG, S. Measuring Sustainable Development: Review of CurrentPractice. Winnipeg: IISD, 1997.

IBGE Indicadores de Desenvolvimento Sustentável Brasil 2002. Estudos e Pesquisas em Geociências, n², Rio de Janeiro, IBGE, 191 p, 2002.

JORGENSEN, S.E. Introduction. In: JORGENSEN, S.E.; COSTANZA, R.; XU, F.L. (Eds.). Handbook of ecological indicators for assessment of ecosystem health. New York: CRC Press Taylor \& Francis Group, 2005.

\section{KRONEMBERGER, D. M. P; CLEVELARIO} JUNIOR, J; DO NASCIMENTO, J. A. S; COLLARES, J. E. R; DA SILVA, L. C. D. Desenvolvimento Sustentável no Brasil: Uma Análise a partir da Aplicação do Barômetro da Sustentabilidade. Revista Sociedade \& Natureza, Uberlândia, v. 20, n. 1, p. 25-50, jun. 2008.
LIRA, W. S. Sistema de Gestão do Conhecimento para Indicadores de Sustentabilidade - SIGECIS: Proposta de uma metodologia. Campina Grande PB. 2008. 178 p. Tese (Doutorado) - Programa de Pós-Graduação em Recursos Naturais, Universidade Federal de Campina Grande. Campina Grande, 2008.

LIRA, W. S; CÂNDIDO, G. A. Análise dos modelos de indicadores no contexto do desenvolvimento sustentável. Revista Perspectivas Contemporâneas, Paraná, v. 3, n. 1, 2008.

MALHEIROS, T. F.; PHILIPPI JR., A.; COUTINHO, S. M.V. Agenda 21 nacional e indicadores de desenvolvimento sustentável: contexto brasileiro. Revista Saúde e Sociedade, São Paulo, v. 17, n. 1, p. 7-20, mar, 2008.

MARANHÃO, N. Sistema de Indicadores para Planejamento e Gestão dos Recursos Hídricos de Bacias Hidrográficas. Rio de Janeiro. 2007. 397 p. Tese (Doutorado) - Universidade Federal do Rio de Janeiro, COPPE. Rio de Janeiro, 2007.

MARTINEZ, R. Indicadores de Sostenibilidad Ambiental y de Desarrollo Sostenible: Estado del Arte y Perspectivas. Série Manuales n 162001 Santiago de Chile CEPAL 116 p. Disponível em: $<$ http://www.eclac.cl/publicaciones/xml/8/9708/ 1c11607e_ind.pdf>. Acesso em 3 set. 2012.

MARZAL, K; ALMEIDA, J. Indicadores de sustentabilidade para agroecossistemas: Estado da arte, limites e potencialidades de uma nova ferramenta para avaliar o desenvolvimento sustentável. Cadernos de Ciência \& Tecnologia, Brasília, v.17, n.1, p.41-59, jan./abr. 2000.

MASERA, O; ASTIER, M; LÓPEZ-RIDAURA, S. Sustentabilidad y manejo de recursos naturales: el marco de evaluación MESMIS. México: Mundi Prensa, GIRA e Instituto de Ecologia, 2000. 109 p.

MORAN, D. et al. Measuring sustainable development - Nation by Nation. Ecological Economics, v.64, n.3, p.470-4, 2008.

\section{ORGANIZATION FOR ECONOMIC COOPE-} RATION AND DEVELOPMENT - OCDE, 1993. Organization for economic cooperation and development: coreset of indicators for environmental performance reviews; a synthesis report by the group on the state of the environment. Paris: OCDE, 1993. 
PHILIPPI JR, A.; MALHEIROS, T. F.; AGUIAR, A. O. Indicadores de desenvolvimento sustentável. In:PHILIPPI JR, A. Saneamento, saúde e ambiente: fundamentos para um desenvolvimento sustentável. Barueri: Manole, 2005. p. 761-808.

PEREIRA, V. S; MARTINS, S. R. Indicadores de sustentabilidade do agroecossistema arroz orgânico com manejo de água contínuo na bacia do Araranguá (SC) mediante aplicação da metodologia MESMIS. Revista Brasileira de Ciências Ambientais, n. $15,2010$.

SALDANHA, E. E. Modelo de Avaliação da Sustentabilidade Socioambiental. Tese de Doutorado - Programa de Pós-Graduação em Engenharia de Produção, UFSC, Florianópolis, 2007.

SILVA, A. M.; CORREIA, A. M. M.; CÂNDIDO, G. A. Ecological Footprint Method: Avaliação da Sustentabilidade no Município de João Pessoa, PB. In: CÂNDIDO, G. A. (Org.). Desenvolvimento Sustentável e Sistemas de Indicadores de Sustentabilidade: Formas de aplicações em contextos geográficos diversos e contingências específicas. Campina Grande, PB: UFCG, 2010, p.236-271.

SPEELMANN, E. N; LÓPEZ-RIDAURÍ, S; COLOMER, N. A; ASTIER, M; MASERA, O. R. Ten years of sustainability evaluation using the MESMIS framework: Lessons learned from its application in 28 Latin American case studies. International Journal of Sustainable Development \& World Ecology, v.14, n.4, p.345-361, 2007.

SOARES, A. B; SILVA FILHO, J. C. L. da; ABREU, M. C. S. de; SOARES, F. de A. Revisando a estruturação do modelo DPSIR como base para um sistema de apoio à decisão para a sustentabilidade de bacias hidrográficas. Revista em Agronegócios e Meio Ambiente, v.4, n.3, p. 521-545, set/dez. 2011.

VAN BELLEN, H. M. Indicadores de Sustentabilidade: Uma Análise Comparativa. 2. ed. Rio de Janeiro: FGV, 2006.

VERONA, L. A. F. A real sustentabilidade dos modelos de produção da agricultura: Indicadores de sustentabilidade na agricultura. Hortic. bras., v. 28, n. 2 (Suplemento - CD Rom), julho 2010.

VIEIRA, P. M. S.; STUDART, T. M. C. Proposta Metodológica para o Desenvolvimento de um Índice de Sustentabilidade Hidro - Ambiental de Áreas Serranas no Semiárido Brasileiro - Estudo de Caso:
Maciço de Baturité, Ceará. RBRH - Revista Brasileira de Recursos Hídricos. v.14, n.4, p. 125-136, out/dez, 2009.

WACKERNAGEL, M. et al. Ecological footprints of nation: how much nature do they use? How much nature do they have? Toronto: Earth Council for the Rio+5 Forum, 1997.

WACKERNAGEL, M; REES, W. Nuestra huella ecológica. Buenos Aires: LOM Ediciones, Colección Ecologia \& Médio Ambiente, 2001. 